Revue d'histoire de l'Amérique française

REVUE D.HISTOIRE DE L'AMÉRIQUE FRANÇAISE

\title{
La pensée politique de Jules-Paul Tardivel
}

\section{Mathieu Girard}

Volume 21, numéro 3, décembre 1967

URI : https://id.erudit.org/iderudit/302690ar

DOI : https://doi.org/10.7202/302690ar

Aller au sommaire du numéro

Éditeur(s)

Institut d'histoire de l'Amérique française

ISSN

0035-2357 (imprimé)

1492-1383 (numérique)

Découvrir la revue

Citer cet article

Girard, M. (1967). La pensée politique de Jules-Paul Tardivel. Revue d'histoire de l'Amérique française, 21(3), 397-428. https://doi.org/10.7202/302690ar d'utilisation que vous pouvez consulter en ligne.

https://apropos.erudit.org/fr/usagers/politique-dutilisation/ 


\section{LA PENSÉE POLITIQUE DE JULES-PAUL TARDIVEL}

\section{Introduction}

Jules-Paul Tardivel est la figure dominante du nationalisme canadien-français de 1867 à 1900. Si jamais l'histoire de la pensée nationaliste au Canada français s'écrit, il recevra, nous en sommes convaincus, une place de choix aux côtés d'un Henri Bourassa et d'un Lionel Groulx.

Nous pouvons considérer Tardivel comme le père de la pensée séparatiste au Québec. En effet, Jules-Paul Tardivel a été le premier Canadien français sous le régime de la Confédération à développer l'idée séparatiste et à défendre l'idéal d'une nation distincte et séparée du Canada anglais.

En présentant Jules-Paul Tardivel, nous voulons dégager de l'œuvre de ce journaliste, vingt-quatre ans rédacteur en chef et propriétaire de la Vérité, ses idées nationalistes.

La pensée de ce dernier est exprimée dans des articles que l'auteur rédigeait alu jour le jour sous le coup de certaines émotions ou sous la dictée d'impérieuses nécessités. On peut retrouver dans certains articles des manquements à la justice et à la charité, une tendance à exercer, sans mandat, et pour la plus grande gloire de Dieu, une sorte de sacerdoce et à s'ériger en souverain juge des actions de ses compatriotes.

Mais ce n'est pas ce qu'il faut retenir. Tout homme a ses défauts et Tardivel n'échappe, en aucune façon, à cette règle. Malgré tous ses défauts et toutes ses insuffisances, le propriétaire et rédacteur en chef de la Vérité a participé à la formation et à l'orientation de la mentalité dans certains milieux du Canada français. Pendant près de 25 ans, son journal circule dans la plupart des presbytères et des collèges ou séminaires de la province de Québec. 
"Or, le Clergé de toute la province lisait le journal de Tardivel; des collèges donnaient en prix, à la fin de l'année scolaire, la collection complète de la Vérité." ${ }^{1}$

Ce que Tardivel apportait dans ces milieux, ce que Tardivel offrait à l'enthousiasme de la jeunesse des collèges et séminaires, c'était un espoir national, celui de voir établir sur les bords du Saint-Laurent une République de la Nouvelle-France complètement indépendante. Toute son œuvre journalistique est imprégnée de cette idée. Toute sa vie témoigne de cette conviction.

C'était pour conserver et protéger cet espoir chez ses compatriotes, c'était pour animer ce patriotisme chez la jeunesse canadienne-française que Tardivel fondait la Vérité le 14 juillet 1881. “Nous n'avons fondé la Vérité que pour souffler sur l'étincelle du patriotisme canadien-français. Lorsque cette étincelle sera devenue incendie, embrasant tous les cœurs; lorsque les chefs Canadiens-français diront hautement au peuple que notre destinée providentielle est de devenir une nation autonome, alors la raison d'être de la Vérité aura cessé, et la Vérité disparaîtra." 2

L'établissement d'un Canada français indépendant sur les bords du Saint-Laurent est pour Tardivel une nécessité religieuse. Il croit à cette possibilité parce qu'il croit à la Providence qui dirige les destinées des nations. Selon lui, le Canada français, réalité catholique avant d'être une réalité politique et économique, est l'instrument qu'a choisi la Providence pour christianiser l'Amérique. Etre séparatiste c'est pour Tardivel être catholique, c'est poser un acte de foi.

Tardivel trouve dans l'histoire des Canadiens français une autre justification à l'idéal séparatiste. Il y voit à chaque moment la main de la Providence qui prépare le peuple canadienfrançais à sa grande destinée. D'ailleurs, la conquête pacifique des territoires frontaliers de la province de Québec par les Canadiens français est une preuve que son rêve n'est pas une folie.

$1 \mathrm{R}$. Rumilly, Histoire de la province de Québec (32 vol., Montréal, 1943-1959), VII : 162.

2 J.-P. Tardivel, "La race canadienne-française", la Vérité, (1897) : 7 . 
Cependant dans ce passé révélateur sur les vues de la Providence sur l'avenir national des Canadiens français, il condamne violemment une bêtise, 1837, la cause directe, dit-il, de 1841 et indirecte de 1867. Que de blâme Tardivel jette à ces "esprits révolutionnaires" qui ont causé un si grand tort à la colonie. La Confédération qui n'a pas donné l'indépendance aux Canadiens français n'a été que la continuation de la politique de Durham qui voulait la disparition des Canadiens français, politique de représailles contre les insurgés de 1837.

Tardivel voit donc dans la Confédération une union législative en germe, "œuvre des sectaires francs-maçons et orangistes" qui ont réclamé l'Union. C'est pourquoi le Canada français doit sortir de ce système politique qui ne peut qu'être temporaire.

Toutefois pour que les Canadiens français atteignent cet idéal national prévu par la Providence, ils doivent, selon Tardivel, réaliser certaines conditions: rester fidèles à Dieu, s'unir en abandonnant l'esprit de parti qui divise les Canadiens français, éviter l'émigration et l'annexion aux Etats-Unis.

Nous présentons aujourd'hui la première d'une série de trois articles sur Tardivel. Dans le premier, nous avons cru nécessaire de faire un bref historique du journal La Vérité. Nous avons voulu ainsi que le lecteur apprenne à connaître l'homme avant de prendre contact avec sa pensée. Dans le second, nous étudierons ses idées sur l'avenir national des Canadiens français. Dans le troisième, nous analyserons les griefs de Tardivel contre la Confédération.

Quand il fonde la Vérité en 1881, Tardivel n'est pas séparatiste. Il le deviendra plus tard. En 1886, Tardivel parlera pour la première fois d'une nation distincte et séparée du Canada anglais. Jusqu'en 1895, sa pensée se précisera dans des articles commentant les événements puisés dans l'actualité. En 1895, Tardivel publiera un roman, Pour la patrie. La parution de ce roman où l'auteur synthétise ses idées séparatistes marque un terme dans l'évolution de sa pensée. De 1895 à 1905, Tardivel tentera principalement de justifier son option nationaliste et de faire partager à ses lecteurs son enthousiasme. 


\section{HISTOIRE SOMMAIRE DE "LA VERRITE"}

Sans contredit, Jules-Paul Tardivel a été, de tous les journalistes canadiens-français de la fin du $\mathrm{XIX}^{e}$ siècle, celui qui s'est valu les critiques les plus acerbes, même les injures les plus abjectes. Rappelons-nous ce passage qui paraissait, encadré de noir, le 16 juin 1885, dans la Minerve de Montréal:

\section{J.-P. Tardivel}

Ceci n'est pas le nom d'un gentilhomme. C'est l'insulteur de feu le docteur Pineault qu'il a appelé homme de mauvaise vie. C'est l'insulteur de tous les français, qu'il a traités d'impies, de révolutionnaires, de traîtres et de sujets de scandales. Nous signalons son nom pour que tous les français le connaissent et puissent lui cracher à la figure quand ils le rencontreront. ${ }^{1}$

Louis-Honoré Fréchette, le poète national, ne ménageait pas non plus son inspiration pour parler de J.-P. Tardivel et de sa Vérité. "Énergumène - scélérat qu'il faudrait fouetter publiquement - fou qu'il serait temps de verrouiller dans un cabanon - feuille confite en dévotion - régenteur d'archevêque être venimeux qui se nomme J.-P. Tardivel — organe ultramonté - blasphème odieux - calomnies infâmes - attaques sans nom - une abomination qui fait dresser les cheveux - fou ou profond scélérat - presse qui s'appelle religieuse mais qui fait le déshonneur de la religion." 2

Certes, J.-P. Tardivel a soulevé les critiques qui dénotaient, chez ceux qui les faisaient, une très grande irritation qui allait parfois jusqu'à la haine. Mais ne pouvait-il pas se consoler à la joie d'avoir reçu le plus beau titre qu'un journaliste catholique pouvait recevoir? Ne l'avait-on pas appelé "le Louis Veuillot du Canada"? (1885) : 3.

1 J.-P. Tardivel, "Hurlements et crachats", La Vérité, 27 juin 2 Ibid. 
Cet homme, qui a reçu les pires injures de ses adversaires, qui était-il? Laissons à Paul Tardivel, son fils, le soin de nous le présenter.

Un canadien de cœur et d'âme, pénétré jusqu'à la moëlle des traditions et des aspirations de notre race, cet écrivain au style clair, précis et d'une impeccable création, était né à Covington, dans le Kentucky, d'un père français et d'une mère anglaise, et, jusqu'à l'âge de dix-sept ans, il ignore même le son des vocables frarç̧ais.

Son père était un montagnard d'Auvergne, originaire des environs cle Billon, où la famille Tardivel compte encore des parents. A dix-huit ans, il suivit en Amérique son compatriote, l'abbé J.B. Lamy, alors simple missionnaire, depuis archevêque de Santa Fe. Sa mère, Isabelle Brent, était la fille de Samuel Brent et d'Anne Vertue, de Cantorbery, Angleterre. Les farnilles Brent et Vertue avaient connu l'aisance, et c'est à la suite de malheurs financiers que Samuel Brent émigra aux Etats-Unis (1827). Il s'établit d'abord à Flat Bush, près de New-York, puis en 1832, se dirigea avec sa famille vers ce qu'on appelait alors le Far West. Tous se fixèrent à quelques milles de l'endroit où s'élève aujourd'hui la petite ville de Mount Vernon, dans l'Ohio. C'était une région peuplée en grande partie de catholiques du Maryland. L'abbé Lamy visitait, en qualité de missionnaire, ces établissements nouveaux. C'est ainsi qu'il vint en relation avec la famille Brent, dont le chef mourut peu après son arrivée dans l'Ohic. Les autres membres de la famille se firent successivement catholiques, le plus jeune même, Julius, entra plus tard dans les ordres sacrés. Les nouveaux convertis s'étaient tellement attachés à leur pasteur que, lorsque celui-ci fut nommé à la cure de Covington, ils l'y suivirent et c'est là qu'au mois de Novembre 1850 le français Claude Tardivel, alorss âgé de vingt-et-un ans et qui exerçait le rude métier de menuisier, épousa l'Anglaise Isabelle Brent. Trois enfants naquirent de ce mariage: Jules-Paul, Anna, aujourd'hui Sœur MaryAloysius, de l'Ordre de Saint-Dominique, à NewYork, et un bébé qui suivit sa mère dans la tombe (1854). 
Le futur directeur de la Vérité n'avait que trois ans - il était né le 2 septembre 1851 - lorsque mourut sa mère. Celle-ci légua ses enfants à sa sœur FrancisIsabella, qui prit spécialement soin de la jeune fille, tandis que Mademoiselle Brent s'occupait plus particulièrement du garçonnet, qui grandit à Dauville, dans l'Ohio, l'une des paroisses desservies par son oncle; et c'est ainsi que sa première éducation fut toute anglaise. Il vécut libre de la vie de l'Ouest, étudiant l'anglais et le latin et travaillant aux champs. Hanté du désir de faire des études classiques, il apprit un beau jour, par Mgr Lamy, l'existence du collège de Saint-Hyacinthe, au Canada. Il y arriva en 1868, ne parlant pas un traitre mot de français. ${ }^{3}$

J.-P. Tardivel entrait donc au collège de Saint-Hyacinthe à l'âge de dix-sept ans, ne connaissant pas le français et très peu de latin. Ses ressources ne lui permettaient pas de passer plus de quatre ans au collège. Les autorités du collège, considérant cette difficulté d'argent et l'âge avancé de l'étudiant, lui permirent de faire deux années en une et ainsi de compléter son cours classique en quatre ans.

En 1872, "philosophe et mathématicien" 4, J.-P. Tardivel retournait aux Etats-Unis avec l'intention de s'y établir. Mais il fut vite déçu. Il se sentit "comme un poisson hors de l'Eau" s.

"Mon séjour de quatre ans au collège de Saint-Hyacinthe et mes vacances passées dans la province de Québec, avaient suffi, mon sang français aidant, pour me franciser complètement le caractère. J'étais dépaysé aux Etats-Unis; j'avais la nostalgie; et au bout de six mois, en janvier 1873, n'y tenant plus, je revins au Canada pour ne plus le quitter ${ }^{6} . "$

J.-P. Tardivel était devenu canadien par nécessité vitale. Il faudrait dire, pour être plus exact, J.-P. Tardivel était devenu Canadien français. Cet homme qui, à dix-sept ans, ne connais-

3 Paul Tardivel, “J.-P. Tardivel”, la Vérité, 29 avril (1905) : 1.

4 J.-P. Tardivel, "Vingt-cinq années de journalisme", La Vérité, 16 avril $(1898): 6$.

5 Ibid.

6 Ibid. 
sait rien de la langue française deviendra un défenseur acharné de tout ce qu'il y a de français au Canada et plus particulièrement dans la province de Québec.

Dès son arrivée, dess amis lui procurèrent un modeste emploi dans un magasin, "mais cela me suffisait, car j'écais au Canada" ?.

Ce travail cependant ne devait pas lui suffire. L'envie d'écrire s'empara vite du néo-canadien. Et, le 23 janvier 1873, paraissait, dans le Courrier de Saint-Hyacinthe, un article intitulé: "Le pape selon les idées protestantes", et signé timidement J. “M. Chagnon m'invita à écrire dans le Courrier, et mon ami Hector Côté se chargea d'écheniller un peu ma prose; car si j'avais déjà à cette époque le cour bien français, mon langage, tant parlé qu'écrit, se ressentait encore terriblement de ma première éducation tout anglaise. ${ }^{8 "}$

Le 15 mars, un autre article paraissait. Ce n'était pas encore cette prolifique production du temps de la Vérité. Enfin, au mois d'avril, le lundi de Pâques, M. Chagnon, directeur du Courrier, engageait définitivement J.-P. Tardivel comme factotum.

Le peu de temps passé au Courrier de Saint-Hyacinthe il quittait M. Chagnon au mois de septembre de la même année - en plus d'initier J.-P. Tardivel au journalisme, lui assurait la présence quotidienne d'un journaliste ancien zouave pontifical: M. Chagnon. Quant on sait avec quelle ardeur J.-P. Tardivel défendit plus tard les Papes et l'Église contre le libéralisme, on peut s'imaginer facilement quelle fascination un zouave devait exercer sur ce jeune journaliste dont le premier article prenait la défense du pape.

Au mois de septembre, Oscar Dunn, qui connaissait Tardivel, l'invite à entrer à la Minerve. "Pauvre vieille Minerve, je te pardonne les dix mois de souffrances que tu m'as fait endurer,

7.J.-P. Tardivel, "Vingt-cinq années de journalisme", La Vérité, 16
avril (1898): 6. 8 Ibid. 
à cause de l'expérience des hommes, et des choses que tu m'as permis d'acquérir ${ }^{9}$."

Le sort en avait décidé ainsi. J.-P. Tardivel devait commencer à apprendre son métier au journal même qui le combattit le plus et auquel il s'attaqua durant toute sa vie de journaliste. À peine entré à la Minerve, J.-P. Tardivel perdait son protecteur Oscar Dunn. Dès ce moment, sa position devint précaire. "Je pouvais être mis à la porte d'une semaine à l'autre ${ }^{10}$."

Cette incertitude dura jusqu'au mois de juillet 1874. Il fut alors engagé comme assistant-rédacteur au Canadien de Québec. "Au mois de juillet 1874, M. Chrysostome Langelier il était bleu à cette époque, seul bleu de sa famille - vint à la Minerve chercher un sous-rédacteur pour le Canadien, de Québec, qu'on venait de réorganiser et de transformer en journal quotidien du matin. Je ne sais trop comment le sort tomba sur moi. Voulait-on se débarrasser de moi à la Minerve, sans me mettre sur le pavé? Je n'en sais. Nous n'étions pas admis aux délibérations des dieux, toujours est-il que M. Langelier me demanda de faire partie du personnel du Canadien avec une légère, très légère augmentation de salaire." 11

Tardivel, marié depuis le 5 février 1874, ne dédaignait pas une augmentation de salaire. Celle, offerte par le Canadien, même très légère, avait dû orienter sa décision. Mais le désir de quitter "ces dieux" le fit accepter sans hésitation. "J'ai à peine besoin de dire qu'on ne fut pas obligé d'insister. Et du jour au lendemain, le 12 juillet, je faisais mes adieux à la Minerve. Ils ne furent pas déchirants!" 12

C'est à Quẻbec, au Canadien, que J.-P. Tardivel connut Israël Tarte. Arrivé au mois de novembre 1874 de Saint-Lin, Tarte était engagé comme rédacteur en chef au Canadien. "Et il fut le maître dès son arrivée." 13

9 J.-P. Tardivel, "Vingt-cinq années de journalisme", La Vérité, 16 avril $(1898): 6$.

10 Ibid.

11 Ibid.

12 J.-P. Tardivel, "Vingt-cinq années de journalisme", La Vérité, 16 avril (1898): 6 .

13 Ibid. 
Tardivel passa sept ans au Canadien avec Tarte, sept années "fort agréables, et même fructueuses" ${ }^{14}$.

“Quoiqu'en dise M. LeBeuf, M. Tarte, à côté de défauts que je ne nie pas, a de grandes qualités. Du moins, il en avait à l'époque. S'il n'en a plus, c'est que les libéraux l'ont gâté complètement. Il m'a toujour's bien traité, je me plais à le proclamer. Depuis cette époque, nous nous sommes éloignés l'un de l'autre. Il affecte de ne pas me reconnaître quand nous nous rencontrons sur la rue." 15

Tardivel ne pardonna jamais à Tarte d'avoir accepté la compagnie des libéraux. Il le combattit avec vigueur, avec dureté, sans relâche, à la moindre occasion. Il y mettait d'autant plus d'ardeur qu'il était convaincu de combattre un transfuge qui avait brisé une amitié de sept ans que Tardivel ne voulait pas oublier. "Mais, pour moi j'ai gardé un excellent souvenir de nos relations; surtout des courtes vacances que nous prenions parfois ensemble dans les montagnes de Valcartier." 16

Même s'il ne l'a jamais dit ouvertement, Tardivel a regretté cette amitié. "Sorti du tourbillon politique qui le gâtait quelque peu même dans ce temps-là, $M$. Tarte était un aimable compagnon et un ami sincère et utile." ${ }^{17}$ Ces paroles écrites en 1898, après dix-sept ans de lutte contre Tarte, prouvent bien que Tardivel gardait à son compagnon du Canadien une amitié que seule sa vocation de défenseur de la vérité et de la justice lui avait interdit d'entretenir. D'ailleurs, tant que Tardivel fut au Canadien, il resta bon ami avec Tarte. Au mois de mai 1881, quand il quitta le journal, Tarte n'y était pour rien.

"Non pas que j'eusse la moindre difficulté avec M. Tarte, mais j'avais en tête l'idée que je pouvais améliorer mon sort. Je songeais à faire une série de livres classiques." 18

\footnotetext{
14 Ibid.

15 Ibid.

16 Ibid.

17 J.-P. Tardivel, "Vingt-cinq années de journalisme", La Vérité, 16 avril (1898): 6 .

18 Ibid.
} 
Ce fut le P. Lacasse, o.m.i., qui le dissuada de cette idée. Lui promettant sa collaboration, le père Lacasse lui fit prendre la détermination de fonder un journal. Et c'est ainsi que parut, le 14 juillet 1881, le premier exemplaire de l'hebdomadaire de J.-P. Tardivel. La Vérité était née.

"Je commençai mon entreprise, selon le conseil du Père Lacasse, et au mois de juillet 1881 le premier numéro de $L a$ Vérité parut." 19

J.-P. Tardivel a consacré sa vie à son journal. Du mois de juillet 1881 au 24 avril 1905 - décès de Tardivel —, le rédacteur de la Vérité a dû faire face à toutes les difficultés; difficultés matérielles qui venaient s'ajouter à une conspiration pour étouffer la Vérité.

Tout d'abord, les choses allaient assez bien. Il m'arriva un certain nombre d'abonnements payés d'avance. Je faisais imprimer la Vérité aux ateliers de l'Evénement, ce qui me coûtait quelque peu cher, il est vrai, mais je n'avais pas à m'occuper de la partie matérielle du journal. Au bout de trois mois, cependant, les ressources commençaient déjà à manquer, et je voyais arriver l'époque où je ne pourrais plus solder régulièrement les factures de mon imprimeur, moment de terrible angoisse dont je me souviens encore, car ce jour-là la situation de la Vérité me fit verser des larmes amères.

Il fallait trouver une autre "combinaison" qui me permit de réduire les dépenses.

Un ami commun me mit en relations avec MM. L. Drouin et Frère, libraires, de Saint-Roch, qui se chargèrent de l'administration de la Vérité.

L'œuvre périclitante s'installa dans un très petit local dont MM. Drouin et Frère pouvaient disposer, une manière de hangar en arrière de leur magasin. J'achetai, avec le peu de fonds qui me restaient, le "caractère" nécessaire pour composer le journal, l'impression devant se faire ailleurs; car il ne fallait pas songer, nous semblait-il, à acquérir une presse. Force nous fut de nous contenter de jeunes apprentis typographes pour faire la composition. Il y eut éco-

19 J.-P. Tardivel, Mélanges (3 vols., Québec 1887-1903), 3: VII. 
nomie de ce chef, mais quelle tâche que la correction des épreuves ! N'importe, mon œuvre me paraissait solidement assise, la joie et la confiance revinrent. Hélas ! instabilité des choses humaines ! A peine le nouveau régime commençait-il à fonctionner un peu régulièrement que nous apprenions qu'un boycott en règle contre la Vérité s'organisait parmi les imprimeurs de Québec. Déjà, sans savoir pourquoi, nous avions vu un tel et un tel refuser d'imprimer le journal. Nous nous étions, à la fin, réfugiés au Chronicle, lorsqu'un beau jour le prote de cet établissement nous fit savoir qu'il imprimait la Vérité pour la dernière fois, et que tous les imprimeurs de Québec, soi-disant pour se protéger, s'étaient entendus pour ne plus accepter l'impression de notre feuille.

Grande consternation au bureau de la Vérité ce jour-là, vous pouvez vous l'imaginer. Mais cette fois je ne pleurai pas. Je résolus de faire un suprême effort pour déjouer cette conspiration contre mon journal. Mais pour cela il me fallait une presse à moi, et j'avais à peine cinquante piastres en caisse. Heureusement, une proche parente, quoique anglaise et habitant un pays étranger, s'intéressait beaucoup à la Vérité. Loin d'être riche, elle m'avait dit, cependant: "Si jamais la Vérité est menacée de mort, faute de quelques cents piastres, ne manque pas de me le faire savoir."

Je lui exposai donc la situation, sans formuler toutefois, aucune demande de secours. Par le retour du courrier je recevais sept cents piastres. Je montai aussitôt à Montréal. MM. Beauchemin et Valois avaient précisément une presse à vendre au prix de sept cent cinquante piastres. Je la payai comptant. C'était un samedi soir, le 10 décembre 1881. La presse fut démontée et expédiée à Québec, le lundi matin. Dès le jeudi, elle était installée dans une chambrette juste assez grande pour la contenir; et la Vérité parut comme à l'ordinaire, au grand ébahissement des adversaires. ${ }^{20}$

Le 24 décembre 1881, Tardivel pouvait écrire en première page: "Messieurs les propriétaires de journaux de Québec ont

20 J.-P. Tardivel, Mélanges, 3: VII-IX. 
dû être surpris de voir paraître notre journal, la semaine dernière. Pourtant ils croyaient avoir pris le moyen le plus sûr de faire tomber notre feuille, ils avaient la douce conviction de nous avoir mis dans des embarras inextricables. Et pour rendre justice à ces messieurs, nous devons avouer que leur petit plan ne manquait pas d'habileté." 21

Quelle consolation pour Tardivel de déjouer le plan de ces premiers adversaires! Consolation d'autant plus grande que les difficultés qu'il avait dû surmonter avaient failli mettre fin prématurément à son œuvre. Consolation toutefois qui devait lui coûter beaucoup d'efforts physiques.

C'était une presse à vapeur que j'avais achetée c'est encore la même qui imprime la Vérité aujourd'hui - et nous n'avions pas de machine à vapeur pour l'actionner. Il fallait imprimer à bras d'hommes, au moyen d'une grande roue d'aire munie de deux manivelles. C'était un ouvrage tellement "forçant" que nous ne trouvions pas facilement les deux hommes voulus pour tourner la roue, même en payant le gros prix. Les journaliers de Saint-Roch et de Saint-Sauveur avaient peur de notre roue comme de la peste. Maintes fois, ne trouvant personne pour tourner la fameuse roue, je dus m'y atteler moi-même, avec M. Odilon Drouin. Excellent exercice et pour le corps et pour l'âme.

Enfin, moyennant finances - une somme assez forte - la municipalité nous permit d'installer une turbine pour faire fonctionner la presse. Mais l'eau manquait bien plus souvent alors qu'aujourd'hui, et le jour la pression n'était généralement pas assez forte pour alimenter la turbine. Alors l'unique moyen d'imprimer la Vérité, c'était d'attendre la fin de l'arrosage des rues et la fermeture des grands ateliers.

En 1890, la Vérité passa par une dernière crise matérielle, la plus forte de toutes, peut-être.

A cette époque, l'honorable M. Mercier était au pouvoir, et il était tout-puissant. La Vérité l'avait appuyé en plus d'une circonstance, mais elle était

21 Tardivel, "Peines Perdues", La Vérité, 24 décembre (1881): 1. 
restée absolument indépendante à son égard et blâmait ceux de ces actes qui paraissaient dignes de blâme. ${ }^{22}$

Tardivel en effet admettait certains points de la politique de Mercier. Mercier avait déclaré, en Chambre, sur l'affaire Riel:

On m'a reproché d'avoir appelé Louis Riel mon frère. J'aime mieux. appeler Louis Riel mon frère que de faire comme certains hommes qui appellent les orangistes leurs frères... Je n'ai jamais eu honte d'un pendu quand il a été pendu pour l'amour de son pays. Je n'ai jamais eu honte d'un Delorimier, d'un Duquet, d'un C'hénier; je n'ai jamais eu honte de mon père fait prisonnier en 1837 parce qu'il aimait son pays.

Il faudrait que je fiusse bien dégénéré pour avoir honte d'appeler Louis Riel mon frère. Louis Riel est mon frère par le sang, comme il est le frère de chacun de vous. Vous avez beau chercher à le renier, cet homme-là, vous serez toujours forcé de vous rappeler la communauté du sang: avant longtemps vous serez obligés, koon gré, mal gré, de défendre sa mémoire. Car souvenez-vous-en, un jour viendra où vos haines politiques disparaîtront, et vous retrouverez alors la place de votre cœur. ${ }^{23}$

Riel:

Tardivel de son côté avait écrit après la condamnation de

Jackson, secrétaire de Riel, a été acquitté comme aliéné sur une preuve beaucoup moins forte que celle qui a été faite en faveur de Riel.

Riel, qui a confié à Jackson, déclaré aliéné, le poste important de secrétaire particulier, est jugé sain d'esprit !

Serait-ce, par hasard, parce que Jackson a des amis à Toronto et que Riel a du sang français dans les veines?

C'en a terriblement l'air; et il faut être naïf et bonasse pour ne pas voir du fanatisme de race dans cet acharnement contre Riel, qui n'est peut-être pas

22 J.-P. Tardivel, Mélanges, 3: IX-X.

23 Robert Rumilly, Histoire de la province de Québec (32 vols., Montréal), $5: 173$. 
sans faute, mais qui ne serait certainement pas aussi grand criminel s'il portait un autre nom et s'il avait fait la guerre à un autre gouvernement. ${ }^{24}$

Similitude de pensée, mais pour des raisons différentes: tandis que Mercier voyait dans l'affaire Riel l'occasion de rallier une majorité et de prendre le pouvoir, Tardivel y discernait l'action de la franc-maçonnerie et de son grand chef, sir John Macdonald, pour frapper les Français. Si les raisons pour appuyer Riel n'étaient pas les mêmes chez Mercier et chez Tardivel, la politique qu'ils préconisaient à la suite de cet événement était identique: l'unité nationale ế l'autonomie provinciale. "Nous tombons d'accord avec M. Mercier sur la question de l'autonomie provinciale." ${ }^{25}$

Tardivel, qui avait lutté pour que le gouvernement provincial remette aux Jésuites les biens qu'ils avaient perdus à la conquête, félicitait Mercier pour sa façon de procéder. "Nous félicitons M. Mercier de procéder dans cette délicate affaire d'une manière aussi régulière..." ${ }^{26}$ Monsieur Mercier, lors d'une visite à Rome, en avait profité pour discuter cette épineuse question avec le Pape. Tardivel s'en était réjoui et souhaitait le succès de Mercier. "C'est pourquoi nous nourrissons l'espoir, avec tous les vrais catholiques de cette province, de voir M. Mercier s'entendre avec le Saint-Siège sur la meilleure manière de restituer toutes ces propriétés qui n'appartiennent à l'État à aucun titre." 27

Pourtant, Tardivel se méfiait de Mercier. N'était-il pas libéral? Il ajoutait en commentaire au programme de Mercier de 1886. "En somme, le manifeste de Mercier n'est pas une œuvre de génie; ce n'est pas un de ces programmes qui remuent, qui enthousiasment, qui soulèvent les foules.

24 J.-P. Tardivel, "Criminels politiques", $L a$ Vérité, 22 août (1885) : 2. 25 J.-P. Tardivel, "Le programme de M. Mercier", La Vérité, 10 juillet (1886) : 6 . 276.

26 J.-P. Tardivel, "Les biens des Jésuites", La Vérité, 31 mars (1888) :

27 J.-P. Tardivel, "Les biens des Jésuites", La Vérité, 31 mars (1888) : 276 . 
Si les libéraux arrivent au pouvoir, ce sera plutôt à cause des fautes de leurs adversaires qu'en vertu de leur propre habilité." 28

Ce n'étaient pas des paroles pour se rendre sympathique auprès de Mercier. Tardivel ne s'en cachait pas. Il disait en 1890: "Si l'on excepte lia défense de l'autonomie provinciale, son programme ne vaut pas mieux que celui de M. Taillon." ${ }^{29}$ Le péché de Mercier était d'être libéral: "Le jour où M. Mercier perdra définitivement sa queue libérale, ce jour-là il doublera ses forces et son prestige." 30

Mais Mercier n'a jarnais perdu "sa queue libérale", et les reproches de Tardivel causaient beaucoup de préjudices à $L a$ Vérité.

Cette indépendance déplut-elle à $M$. Mercier luimême et résolut-il de créer des embarras à la Vérité? Je ne saurais l'affirmer. Toujours est-il que certains intrigants, qui tenaient de près au cabinet, réussirent à persuader MM. L. Drouin et frère que leur commerce de librairie irait mieux, qu'ils recevraient de fortes commandes du gouvernement s'ils cessaient d'administrer la Vérité et même de lui donner l'hospitalité.

Mis au courant de la situation, et ne voulant nuire en rien à $M M$. Drouin et frère, à qui je devais beaucoup de reconnaissance pour l'accueil qu'ils m'avaient fait au moment de mes premiers embarras, je me rendis immédiatement à leur désir. La séparation se fit très amicalement, et j'eus aussitôt le regret de constater que mes prévisions, dont j'avais fait part à $M$. Louis Drouin, s'étaient réalisées à la lettre: Ia Vérité partie de leur établissement, les commandes du gouvernement, au lieu d'augmenter, cessèrent tout à fait.

Ce fut avec crainte et tremblement que j'entrepris l'administration du. journal. Mes amis m'avaient

\footnotetext{
let $(1886): 6$.

29 J.-P. Tardivel, "Le discours-programme de M. Mercier", La Vérité, 24 mai (1890): 3.

30 J.-P. Tardivel, "Conservateurs-nationaux et libéraux", La Vérité, $1^{\text {er }}$ septembre $(1888): 51$.
}

28 J.-P. Tardivel, "Le programme de M. Mercier," La Vérité, 10 juil- 
tant répété que je n'entendais rien aux affaires, que j'étais bon tout au plus à la rédaction, que j'avais fini par le croire. C'était, du reste assez vrai: je commençai par un faux pas.

Dans l'espoir d'améliorer la situation du journal, toujours très précaire, je voulus agrandir le champ de mes opérations, en créant une imprimerie d'ouvrages de ville. Grâce à la générosité de quelques amis, à qui j'avais fait part de mon projet, je montai, à Saint-Roch, à des frais assez considérables, un atelier d'imprimerie générale.

Me voilà donc directeur, rédacteur en chef et administrateur du journal, et, par surcroît, chef d'imprimerie.

C'était une folle entreprise.

Heureusement saint Joseph, qui a toujours été le véritable "homme d'affaire" de la "Vérité", me fit comprendre, à temps, qu'à vouloir conduire deux besognes à la fois, je les gâcherais inévitablement toutes deux. Il m'inspira l'idée de me faire petit, au lieu de vouloir me faire grand. J'abandonnai donc, le plus tôt possible, mais non sans d'assez lourdes pertes, ma téméraire entreprise d'agrandissement qui eût fini par m'entraîner à la ruine complète; et je résolus de m'occuper à l'avenir exclusivement du journal. Je transportai bureaux et ateliers à la maison que, grâce à la générosité d'autres amis, je venais d'acquérir sur les hauteurs de Sainte-Foye près de la ville. J'installai la "Vérité" dans la cave et en fit une œuvre domestique, très modeste, mais solide.

Depuis le mois de février 1891 que la "Vérité" est ainsi "domestiquée", tout va bien, sous le rapport matériel. Plus de crises financières périodiques, plus d'inquiétudes, plus de billets à négocier - les gérants de banque ne me connaissent seulement pas de vue - plus de créanciers à pacifier. Le journal durera nécessairement aussi longtemps que le bon Dieu me laissera la santé. Les "coups de vent", les "affaires montées", le mauvais vouloir de celui-ci ou de celui-là n'ont plus de prise sur la "Vérité". En se faisant petite, et en se retirant dans une cave, fort convenable du reste, elle est à l'abri de toutes les intempéries. 
Par contre, si la "Vérité", dans sa cave, est à l'abri des tempêtes, l'air tranquille et parfois un peu humide qui l'enveloppe l'empêchera toujours de grandir. Elle sera - nécessairement, jusqu'à la fin - l'œuvre modeste que l'on connaît. Et si elle donne juste de quoi vivre, ce qu'elle ne faisait pas lorsqu'elle poussait en plein vent, elle ne me permettra certes jamais de réaliser cent écus. J'ai eu beau faire des économies, en accumulant à peu près tous les emplois, depuis celui de directeur jusqu'à celui de chauffeur de la machine à vapeur, jamais le budget de la "Vérité" ne se solde par le moindre surplus. Le journal ainsi organisé, me donne, avec la tranquillité nécesssaire à tout travail intellectuel, le pain quotidien. C'est d'ailleurs tout ce qu'il faut demander au Père céleste; et tous les jours je remercie saint Joseph de m'avoir inspiré ce plan, si simple en apparence, mais que je n'aurais pas pu trouver par moi-même. ${ }^{31}$

Tardivel a réussi à joindre les deux bouts grâce à l'aide de ses amis. Parmi ceux-ci il y avait des prêtres, et Tardivel ne s'en cachait pas. Tarte, dans un article que rapporte Tardivel, l'accusait de ce soutien: "Il est de notoriété publique qu'une division profonde existe dans les rangs du clergé, dans l'épiscopat. Des évêques ont cessé d'avoir sur les prêtres des diocèses l'autorité et l'influence. Des journaux ont été fondés et sont soutenus avec l'argent du clergé, qui, lorsque l'occasion s'en présente, combattent ouvertement les enseignements des chefs constitués de la hiérarchie catholique et n'épargnent rien pour détruire le respect qui leur est dû. Les souscriptions en faveur de ces journaux sont classées au rang de "bonnes œuvres". En plus d'un quartier, le soin des pauvres, le denier de Saint-Pierre, la Propagation de la foi et la Sainte-Enfance passent après ces "œuvres"." 32

La "Vérité" se défendait bien d'avoir été fondée par le clergé. "Les journaux auxquels M. Tarte fait allusion sont l'Etendard et La Vérité; il les nomme un peu plus loin. Or, parlant pour nous-mêmes, nous déclarons que notre journal n'a

31 J.-P. Tardivel, Mélanges, 3 : X-XII.

32 J.-P. Tardivel, Mélanges, 3 : 131. 
pas été fondé avec l'argent du clergé. Nous avons mis dans cette œuvre un petit patrimoine et quelques faibles épargnes, sachant bien que ce placement ne nous rapportait jamais un sou d'intérêt, mais fermement convaincu que nous faisions, en effet, une "bonne œuvre", œuvre que M. Tarte a été le premier à saluer." 33

Mais Tardivel ne niait plus son encouragement: "Si quelques membres du clergé, des dignitaires ecclésiastiques ont bien voulu nous aider, depuis la fondation de notre feuille, c'est qu'eux aussi ont jugé notre œuvre bonne; et certes ils n'ont pas de compte à rendre aux écrivains de l'Evènement." ${ }^{34}$

J.-P. Tardivel, "parlant pour nous-mêmes", avouait neuf ans plus tard, en 1893, d'une façon moins équivoque cet encouragement que son journal avait reçu. Et il donnait, à propos de l'Etendard, des chiffres qui montrent que Tarte avait vu juste.

Ainsi, pour ne parler que de la ville et du district de Montréal, sur lesquels j'ai des renseignements plus complets, je puis dire que, d'après des informations puisées aux meilleures sources, les souscriptions versées par les catholiques de la ville et du district en question pour la fondation et le support de l'Etendard pendant les dix années de son existence, se sont élevées à près de 100,000 (cent mille) piastres ou 500,000 francs. Avant lui, le NouveauMonde avait coûté presque autant; et après lui, vers 1895, La Croix, en 2 ou 3 ans, reçut en purs dons pas moins de 25,000 (vingt-cinq mille) piastres ou cent vingt-cinq mille francs.

A cela, pour se former quelque idée des sacrifices qu'ont faits nos concitoyens catholiques, il faudrait ajouter ce qui a été accompli pour la même cause sur divers autres points du pays; il faudrait ajouter, en particulier, l'aide accordée à la "Vérité" ou à son directeur, comme j'ai déjà eu occasion de le dire, aide se montant à plusieurs mille piastres, provenant en bonne partie des citoyens de la ville et du district de Montréal.

33 J.-P. Tardivel, Mélanges, 3 : 131.

$34 \mathrm{Ibid}$. 
Jugez par là que serait la presse catholique parmi nous si l'appui moral que Pie IX et Léon XIII veulent qu'on lui accorde ne lui avait pas manqué. ${ }^{35}$

J.-P. Tardivel trouvait amer le peu d'encouragement que son journal recevait de ses concitoyens. Montréal était généreux pour soutenir les journaux catholiques de son district... et même pour la Vérité de Québec. Mais que penser des abonnés de la Vérité.

Depuis le mois de février 1891, ai-je dit, les affaires matérielles du journal "vont bien". Cela n'est vrai que "relativement parlant"; car les épreuves inhérentes à toute œuvre de journalisme catholique ne me manquent certess pas, et ne m'ont jamais manqué un seul instant.

Par exemple, combien de gens "encouragent" la "Vérité" en s'y abonnant, mais qui "oublient" ensuite de payer leur abonnement, malgré les appels réitérés de l'administration, laissant accumuler des arrérages dont le chiffre, exagéré pourtant, étonne encore moins parfois que le caractère du retardataire. Il m'est toujours dû, depuis des années, des arrérages d'abonnement s'élevant à plus d'un millier de piastres. Que de fois il m'a fallu rayer les noms des mauvais payeurs de mes listes, car je préfère toujours perdre une somme connue que de risquer un procès. Si j'avais en caisse toutes les sommes ainsi perdues, sommes légitimement gagnées, ce me semble, je ne serais pas tant aux prises avec la pauvreté. Puis, combien, payant irrégulièrement, s'imaginent "avoir payé", et contestent l'état de compie qu'on leur envoie. ${ }^{36}$

A ces difficultés d'argent vinrent s'ajouter les procès. Frappant sans arrêt au nom de la justice, Tardivel n'a pas manqué de soulever la colère de ses adversaires. Mais ceux qui le poursuivaient n'étaient pas sans reproches. C'est ce qui sauva Tardivel.

Dans l'ordre des épreuves "matérielles" mentionnons les procès que j'ai eu à soutenir. Plusieurs, il est vrai, ne furent que de mauvaises plaisanteries, 
comme la poursuite que M. Tarte m'intenta un jour pour me demander de lui verser la modeste somme de trente-cinq mille dollars; et celle de l'honorable M. R. Laflamme qui se serait contenté, lui, de cinq mille. Ces deux affaires n'eurent aucune suite.

M. Fréchette (notre poète "national"!) s'avisa de me poursuivre, tant au civil qu'au criminel; mais le brave homme s'aperçut que c'était là un jeu qui se joue à deux, et que ceux qui habitent des maisons de verre ont tort de vouloir se défendre à coups de pierres. Une contre-poursuite, au civil et au criminel, mit fin à l'affaire Fréchette dans le temps de le dire.

En décembre 1891, voilà M. Mercier qui lui aussi, me cita devant les tribunaux, pour "libelle séditieux", s'il vous plaît. J'avais demandé une enquête sur certains bruits qui circulaient dans le monde politique. Ce pauvre Mercier, se sentant aller au fond, était devenu exaspéré au dernier point. Il voulait sérieusement me fourrer au bagne pour un temps indéterminé. Si frivole parut l'accusation que le magistrat Chauveau refusa de lancer un mandat d'amener contre moi; mais son collègue, M. Murray, se montra plus complaisant, et je fus conduit au palais de justice par deux énormes agents de la police provinciale, au grand désespoir de mes enfants.

L'affaire avait un côté très comique, mais elle aurait pu tourner au tragique, car M. Murray semblait bien déterminé à m'envoyer devant la cour d'assises, et le représentant du Procureur général, feu M. Guillaume Amyot - que le bon Dieu l'ait dans son saint Paradis! - se montra singulièrement acharné contre moi. Mais le 16 décembre, M. le lieutenant-gouverneur Angers donna son fameux coup de balai, et avec la chute du cabinet Mercier, mon affaire, ainsi que bien d'autres, tomba à l'eau.

Le seul de mes procès qui m'ait réellement peiné, tout en me causant de lourds dommages, fut l'affaire Sauvalle.

M. Marc Sauvalle, français de naissance, était l'un des principaux rédacteurs du "Canada-Revue", de Montréal, qui, il y a une dizaine d'années, menait une campagne de scandale contre le clergé catholique. 
Sur ces entrefaites, le "True Witness" annonça que M. Sauvalle faisait baptiser ses enfants par un ministre méthodisie. Je crus, de bonne foi, M. Sauvalle réellement méthodiste, et je lui appliquai cette épithète. J'appris bientôt après qu'il était officiellement catholique. Je publiai aussitôt et spontanément une rectification; mais je ne pus m'empêcher de dire que le cas de M. Sauvalle "catholique" faisant baptiser ses enfants par un ministre hérétique était plus grave que je ne l'avais supposé.

M. Sauvalle me poursuivit, et bien qu'il ne pût prouver aucun dommage réel, $M$. le juge Jet.é ne tint aucun compte de ma rectification et me condamna au plein montant des dommages-intérêts réclamés par mon adversaire: deux cents piastres, et à tous les frais, une affaire de quatre ou cinq cents piastres en tout.

Sur l'avis de plusieurs des meilleurs jurisconsultes du pays, je portai l'affaire en appel. Mais il était écrit, il faut croire, que je n'obtiendrais pas justice des hommes; et la cour d'appel, présidée par sir Alex. Lacoste, et composée en outre, de MM. Bossé, Blanchet, Hall et Wurtele ratifia, à l'unanimité, le jugement de M. Jetté.

Ainsi j'eus à débourser - ou plutôt mes amis voulurent bien débourser pour moi - car alors, comme aujourd'hui, je n'avais aucun fonds de réserve la somme de huit cents piastres, pour un mot inexact, il est vrai, mais retiré aussitôt et qui, en réalité, représentait M. Sauvalle sous un jour trop favorable! Sans la générosité de mes amis, j'étais un homme ruiné. Huit cents piastres, c'était beaucoup plus que mon revenu annuel d'alors. Qui soutiendra qu'un tel jugement était proportionné à la faute?

Et songez aux efforts d'esprit qu'il m'a fallu déployer, depuis lors, pour éviter de nouvelles poursuites, tout en menant un peu résolument la lutte. ${ }^{37}$

J.-P. Tardivel ne tolérait pas qu'on lui défendît de combattre le mal où qu'il soit. C'était son devoir de défendre le droit et la vérité. Le journal est une invention diabolique: "La presse est aux intelligences, ce que les engins de guerre moder-

37 J.-P. Tardivel, Mélanges, 3: XIII-XV. 
nes sont aux corps: Un moyen de tuer beaucoup et de loin." 38 Cette arme au service du mal, il fallait la retourner contre elle-même. Le chrétien doit se servir du journal pour lutter contre le journal: "Faut-il conclure que la presse n'opère que le mal, qu'elle soit impuissante pour le bien? Non, assurément. De même que le canon Krupp, la mitrailleuse et la torpille peuvent servir à la défense d'une cause sainte, à repousser d'injustes invasions, de même aussi le journal peut être un instrument de bien s'il est employé à défendre la vérité contre le mensonge et l'erreur. Et de même que le soldat qui combat pour le droit peut et doit se servir des armes les plus perfectionnées, de même aussi c'est une obligation pour le chrétien de s'emparer de la presse pour mieux défendre l'Église et la société." 39

Son intention était bonne, Tardivel se croyait frustré du droit d'attaquer les hommes. Mais les autres, en avaient-ils le droit? "Embusquées dans le journal, l'envie, la jalousie, la médisance, la calomnie, la haine, la vengeance, lancent leurs traits empoisonnés sur les réputations les plus pures, sur les caractères les plus nobles, sur les institutions les plus respectables, sur les choses les plus saintes. Insinuations malveillantes, "faitsdivers" perfides, correspondances mensongères, articles diffamatoires, voilà les armes redoutables que le journal met à la disposition des méchants." 40

Voilà pour le côté matériel de son œuvre.

Du côté "moral", l'histoire de la Vérité est encore plus mouvementée peut-être.

Et d'abord, dans les premiers temps, lorsque je manquais entièrement de ressources, deux fois je fus soumis à une rude tentation. Des hommes politiques m'offrirent de l'argent pour soutenir mon œuvre. Il y eut pourparlers; mais à la dernière minute, je constatai qu'en retour des fonds offerts on exigerait des services politiques.

Divers gouvernements, tant d'une couleur que de l'autre, m'ont aussi offert des "annonces" et des

38 J.-P. Tardivel, Mélanges, $1: 109$.

39 J.-P. Tardivel, Mélanges, 1: 109.

40 J.-P. Tardivel, Mélanges, 1: 110-111. 
"impressions"; je les ai constamment refusées, ce qui n'a pas empêché mes adversaires de m'accuser, plus d'une fois, d'être inféodé, tantôt au parti libéral, tantôt au parti libéral-conservateur. ${ }^{41}$

J.-P. Tardivel parle ici de libéral-conservateur en parlant du parti de Chapleau. Le vrai conservateur est un vrai catholique ou vice versa un vrai catholique est un vrai conservateur: "Un vrai conservateur est un homme qui travaille à la conservation, non de certaines lois, non de telle ou telle institution, non du "statu quo", mais de la nation. Et comment doit-il travailler? Par la justice? "Justitia elevat gentes"... Or, tout homme qui est parfaitement pénétré de l'esprit de Jésus-Christ, tout homme qui est sincèrement chrétien, aime la justice. Donc, tout bon catholique est vraiment conservateur dans la plus large acception du mot, et plus il s'éloigne de la Vérité du Christ, moins il est conservateur." ${ }^{42}$ Chapleau qui s'était associé à Sénécal ne pouvait, ainsi que le parti dont il était le chef, être considéré comme conservateur. "Les conservateurs sont ceux qui voudraient que leurs intérêts matériels fussent défendus sans employer l'Évangile... Non, jamais nous ne consentirons à nous courber devant le centre conservateur... il détruit la sève et cause la mort certaine de la société." ${ }^{43}$ Et une autre fois: "Nous avons toujours dit que ces bons "bleus" ou soidisant conservateurs sont de fieffés hypocrites quand ils nous parlent de leur grand respect pour les enseignements de l'Église et de leur horreur invincible pour le libéralisme." 44

Tardivel ne pouvait pas accepter l'aide de ces partis. Sa conscience le lui défendait. "Mais ne demandant rien à aucun parti, ni à aucune faction, ma conscience me rend le témoignage que j'ai toujours été vraiment libre - et j'espère bien l'être toujours - à l'égard des hommes, et cela me suffit.

Jamais je n'ai accepté une aide quelconque de ceux qui sollicitaient des services en retour. Les personnes qui, en diverses

41 J.-P. Tardivel, Mélanges', 3 : XVI.

42 J.-P. Tardivel, Mélanges, 1: 308. 239.

43 J.-P. Tardivel, "Le Coriservatisme", La Vérité, 25 février (1888) :

44 J.-P. Tardivel, "Principes bleus", La Vérité, 7 avril (1888) : 285. 
occasions, ont soutenu mon œuvre, parfaitement désintéressées elles-mêmes, ne m'ont jamais demandé qu'une chose: la défense, libre de toute entrave, des intérêts généraux du pays.

J'ai poussé cet amour de la saine indépendance jusqu'au point de cesser aussitôt que j'ai pu le faire, de publier la moindre annonce, la moindre réclame commerciale." 45

Tardivel ne croyait pas pouvoir concilier "esprit de parti" et "esprit catholique". Celui que l'esprit catholique anime travaille à faire ce que Dieu exige de lui: il cherche d'abord le royaume de Dieu et sa justice. Que le premier ministre et le chef d'opposition soient contents ou mécontents, que la "Minerve" applaudisse ou qu'elle condamne, que la "Patrie" rage ou qu'elle jubile peu lui importe. Il craint Dieu et n'a pas d'autre crainte. Il va droit son chemin; il ne vend pas sa conscience pour de l'or ou un honneur; il n'achète pas la conscience de son voisin; il blâme ce qui est blâmable; il approuve ce qui est bien; en un mot, il se montre chrétien dans la vie sociale et dans le monde politique.

Tel est le sens catholique, l'esprit catholique, unique remède contre l'esprit de parti.

"L'esprit catholique vient de Dieu, il vivifie et unit: l'esprit de parti vient de l'homme et du démon, il désunit et tue." 46

Ces paroles ne pouvaient pas être plus catholiques. On imagine tout le clergé se lever et applaudir à cette profession de foi. Pourtant, Mgr A. Taschereau n'aimait pas la "Vérité".

Dès le début, j'eus le chagrin - chagrin plus grand que je ne puis dire - de constater que la "Vérité" déplaisait beaucoup à $\mathrm{Mgr}$ Taschereau, archevêque de Québec.

Le premier froissement eu [sic] lieu à propos du travail du dimanche sur le chemin de fer de la Rive nord, alors propriété du gouvernement provincial, aujourd'hui tronçon du Pacifique Canadien.

45 J.-P. Tardivel, Mélanges, 3 : XVI-XVII.

46 J.-P. Tardivel, "Esprit catholique et sens catholique", La Vérité, 20 février (1886) : 2 . 
A la demande même de Sa Grandeur, j'avais entrepris contre ce scandale une campagne que je menai rondement pendant quelques semaines. Tout à coup, je reçus de son secrétaire l'ordre de discontinuer. J'obéis; mais je voulus savoir de Monseigneur luimême si j'avais mal conduit la lutte. Sa Grandeur m'accorda une longue audience. Je n'appris pas ce que je désirais savoir. Je constatai seulement que Mgr Taschereau était absolument opposé à la "Vérité", même au journalisme catholique - tel qu'on l'entend généralement.

Pour Sa Grandeur, dont j'ai saisi parfaitement les paroles, paroles bien claires, du reste, et sur le sens desquelles il m'a semblé impossible de me méprendre, les journalistes laïques n'ont pas à s'occuper de la défense de l'Eglise et de sa doctrine, n'ont pas pour mission de propager la vérité catholique et de combattre les erreurs doctrinales. "Laissez aux gens d'églises, me dit-elle en propres termes, les choses de l'Eglise, et bornez-vous à traiter les questions de votre ressort: politique, agriculture, colonisation, etc." 47

Mgr Taschereau, dans sa lettre du 11 octobre 1881 pour ordonner à Tardivel de cesser ses articles sur le travail du dimanche, disait assez sèchement au rédacteur de la "Vérité" de ne pas se mêler des affaires de l'Église.

Monsieur le rédacteur,

Je suis chargé par Sa Grandeur Monseigneur l'Archevêque de vous écrire que c'est son désir formel de vous voir cesser vos articles au sujet du travail du dimanche, sur la ligne du chemin de fer du Nord. Monseigneur l'Archevêque, s'il juge nécessaire, se mettra en rapport avec qui de droit, pour régler cette question.

J'ai l'honneur d'être, monsieur le rédacteur, avec une parfaite considération.

Votre très humble serviteur,

C. A. Collet, ptre, secrétaire. ${ }^{48}$

47 J.-P. Tardivel, Mélanges, 3 : XVII-XVIII.

48 J.-P. Tardivel, Mélanges, $1: 108$. 
Que s'était-il passé depuis le mois de septembre? En effet, le 5 septembre, en réponse à une lettre du rédacteur de la Vérité, Mgr Taschereau s'était déclaré sans équivoques en faveur de la lutte que poursuivait Tardivel pour faire cesser le travail le dimanche sur la ligne du chemin de fer du Nord. "Je désire de tout cœur voir cesser les trains du dimanche sur le chemin de fer Q.M.O. et O. Ce désir, je l'ai manifesté, dès le 2 mars dernier, dans une lettre adressée à l'honorable M. Chapleau." 49

M. Chapleau avait-il quelque chose à faire dans ce changement de position aussi subit qu'incompréhensible? On sait qu'à cette époque, le gouvernement faisait tout en son pouvoir pour vendre le chemin de fer du Nord. Croyait-on dans les milieux parlementaires que cette campagne pour faire cesser le travail du dimanche pouvait nuire à la vente? Avait-on fait des pressions sur Mgr Taschereau pour faire cesser cette campagne?

Tardivel pouvait se poser sérieusement toutes ces questions. Mgr Fabre et Mgr Laflèche avaient donné leur approbation à la campagne et n'avaient jamais cessé de l'approuver. ${ }^{50}$ Mais le rédacteur de la "Vérité" ne pouvait exprimer publiquement ses doutes et son désaccord avec son évêque et sur la conduite de ce dernier.

Pourtant, il ne pouvait rester silencieux. Quelle colère a dû s'emparer de Mgr Taschereau quand il lut le 20 octobre, seulement neuf jours après sa lettre à Tardivel, dans la Vérité, l'article intitulé: "La presse catholique".

"Plusieurs personnes, parfaitement sincères et bien intentionnées, voient d'un très mauvais œil la presse catholique et les luttes qu'elle est obligée de soutenir pour la défense des saines doctrines et pour repousser les attaques plus ou moins perfides et déguisées des ennemis de l'Église. Ces personnes affirment que ce n'est pas aux laïques à intervenir dans les questions où la religion est intéressée, que le clergé doit seul défendre l'Église, et que les journalistes ne devraient s'occuper

49 J.-P. Tardivel, Mélanges, 1: 105.

50 J.-P. Tardivel, Mélanges, 1: 106. 
que des affaires purement matérielles, ne devraient traiter, dans leurs colonnes, que des questions de finances, de voies ferrées, de canaux, d'agriculture, etc." 51

Ces paroles, il ne fait aucun doute, étaient adressées à Mgr l'Archevêque de Québec.

J'eus beau faire remarquer à Monseigneur, aussi respectueusement que possible, que l'Eglise, le SaintSiège, le Pape, les Evêques, les ordres religieux, les vérités catholiques et les vertus chrétiennes étant sans cesse attaqués, directement ou indirectement, par nombre de journaux, la défense, me semblait-il, devait se produire également dans la presse. Je ne réussis pas à convaincre Sa Grandeur de l'utilité des journaux catholiques en général, et encore moins de la "Vérité" en particulier. "Si en passant sur le chemin, me dit-elle, un gamin vous insulte, vous ne vous amusez pas à lui répondre, n'est-ce pas? Eh bien! c'est ainsi qu'i]. faut faire à l'égard de la mauvaise presse." Je me permis de faire voir en quoi cette comparaison me semblait manquer de justesse; mais tout fut inutile: Mgr Taschereau resta après comme avant l'entrevue, résolument opposé à l'œuvre de la presse catholiçue.52

Dans la conclusion de l'article du 20 octobre, Tardivel ne cachait pas son désaccord avec son évêque. "Voilà pourquoi les laïques ont le droit de traiter les questions qui intéressent la religion, car tout ce qui intéresse la religion intéresse l'État. Dans la pratique, sans doute, il faut de la prudence, il faut que les laïques obéissent à leurs supérieurs ecclésiastiques et s'en rapportent à eux quand il s'agit de tel ou tel principe. MAIS PERSONNE NE PEUT LEUR CONTESTER LE DROIT DE PROCLAMER ET DE IIEFENDRE LES DOCTRINES DE L'ÉGLISE." 5354

Ce n'était pas là la fin des épreuves de Tardivel. "Lors des pénibles débats qui eurent lieu dans la presse au sujet le la

51 J.-P. Tardivel, Mélanges, 1 : 113.

52 J.-P. Tardivel, Mélanges, 3 : XVIII.

53 C'est nous qui soulignons.

54 J.-P. Tardivel, Mélanges, 1: 116. 
division du diocèse des Trois-Rivières, je pris fait et cause pour l'évêque des Trois-Rivières et le commissaire apostolique. Pouvais-je faire autrement? On voulait diviser le diocèse des TroisRivières malgré Mgr Laflèche, et POUR LE PUNIR ${ }^{55}$ - on le disait ouvertement dans certains cercles. Je savais le représentant du Saint-Siège, Mgr Smeulders, tout à fait opposé à cette division; il l'avait déclaré ouvertement." 56

Tardivel croyait apercevoir, au fond de cette affaire, de basses et inavouables intrigues, une influence occulte des loges maçonniques. Les intérêts généraux du pays, comme la justice envers l'évêque des Trois-Rivières et la population de son diocèse et l'honneur du Saint-Siège paraissaient en cause selon Tardivel. "J'entrai donc en lice contre le ban et l'arrière-ban du libéralisme, pour en montrer la mauvaise foi et les intrigues." ${ }^{57}$

D'ailleurs, Tardivel affirme que s'il n'avait pris parti pour Mgr Laflèche et le commissaire apostolique, s'il était resté "neutre" en cette circonstance, "j'en éprouverais aujourd'hui, et jusqu'à la fin de ma vie, le plus cuisant remords." 58

Cette lutte contre la division du diocèse des Trois-Rivières lui valut une nouvelle humiliation de la part de Mgr Taschereau qui semblait partisan de la division de ce diocèse.

“Touchons encore cependant à quelques épreuves, dans l'ordre moral, avant d'aborder un chapitre plus agréable, celui des consolations." 59

En 1888-89, des amis de Tardivel, sans la moindre sollicitation de sa part, lui fournirent généreusement les moyens de faire un voyage de sept mois en Europe. Tardivel a beaucoup apprécié ce voyage, mais il l'affirme lui-même, ce fut "l'occasion d'une des plus fortes épreuves de ma vie". ${ }^{60}$

$55 \mathrm{C}$ 'est l'auteur qui souligne.

56 J.-P. Tardivel, Mélanges, 3 : XXII.

57 J.-P. Tardivel, Mélanges, 3 : XXIII.

58 Ibid.

59 J.-P. Tardivel, Mélanges, 3 : XXXII.

60 Ibid. 
Tardivel, quoique porteur d'une lettre de recommandation, ne put obtenir d'audience avec le Pape. Il voyait là encore l'œuvre de ses adversaires qui avaient réussi à lui faire fermer les portes.

"Mais de toutes les épreuves de ma vie de journaliste, la plus amère, je dois l'avouer, m'arriva au commencement de 1887." 61

Pendant les troubles de 1885, au Nord-Ouest, et pendant une bonne partie de l'agitation politique qui en fut la conséquence, Tardivel, de son propre aveu, n'écrivit rien sur cette brûlante question sans consulter Mgr Laflèche. Il voulait profiter de l'expérience de l'évêque des Trois-Rivières sur les questions qui se rapportaient aux sauvages et aux métis. Mgr Laflèche avait été, pendant de longues années, missionnaire dans les régions du Nord-Ouest.

Or, Mgr Laflèche était fermement persuadé que, lors du soulèvement de 1885 , le grand coupable était le gouvernement fédéral, qui avait négligé de s'occuper des griefs des métiss. L'exécution de Riel était, à ses yeux, une concession déplorable à la haine orangiste; et pour lui, comme pour nous tous, le gouvernement Macdonald méritait la déchéance. En un mot, Mgr des Trois-Rivières fut pendant longtemps ouvertement favorable au mouvement dit national. Il soutenait et encourageait l'"Etendard" et la "Vérité", même après que tant d'autres eurent abandonné et anathématisé ce beau réveil du patriotisme canadien-français, lequel, bien dirigé, aurait pu régénérer notre race. ${ }^{62}$

Cependant, vers la fin de 1886, la Minerve et d'autres journaux ministériels commencèrent à répéter que le mouvement national était condamné même par Mgr Laflèche, comme révolutionnaire. Pour en avoir: le cœur net, l'honorable M. Trudel, directeur de l'Étendard, et J.-P. Tardivel résolurent de poser carrément la question à Mgr Laflèche, à savoir s'il condamnait, oui ou non, le mouvement national.

61 J.-P. Tardivel, Mélange:3, 3 : XXXIV.

62 Ibid. 
Le 12 novembre 1886, Tardivel rejoignait, à Trois-Rivières, deux prêtres, représentant l'Étendard, pour rencontrer $\mathrm{Mgr}$ Laflèche. Tardivel affirme que même s"'il n'était plus l'approbateur décidé du mouvement national qu'il avait été auparavant" "63, Mgr Laflèche "nous déclara formellement qu'il ne condamnait pas le mouvement politique dit national". ${ }^{64}$

Les journaux ministériels continuant à accuser la Vérité d'être condamnée comme révolutionnaire par tous les évêques, même Mgr Laflèche, Tardivel publia, avec la permission de l'évêque des Trois-Rivières, le 18 novembre 1886, un résumé de l'entrevue du 12 novembre.

Or, le 17 janvier 1887, Mgr Laflèche écrivait à M. Montplaisir une lettre rendue aussitôt publique où il condamnait formellement, avec sévérité même, le mouvement national et ceux qui y prenaient part et où il recommandait le maintien au pouvoir du cabinet Macdonald.

“Jamais, je n'éprouvai, jamais, il me semble, je ne pourrai éprouver en ce monde une douleur semblable à celle qui me serra le cœur à la lecture de cette lettre. Et, à partir de ce jour, et jusqu'à la fin, MIgr Laflèche fut l'adversaire déclaré de l'Étendard et de la Vérité, nous dénonçant publiquement et sans miséricorde." 65

Et Tardivel d'écrire, vingt-cinq ans plus tard, en pensant à cet événement: "Quelle perte notre pays a faite en perdant son Bourget!" 66

Tardivel n'a pas eu seulement des épreuves; des encouragements, très précieux, ne lui ont jamais fait défaut. Les nombreux secours matériels que reçut le rédacteur de la Vérité d'amis dévoués lui ont permis, outre de faire un voyage en Europe en 1888-89, de faire vivre son journal dans ses débuts difficiles. Tardivel aurait aimé recevoir du Pape lui-même la

63 J.-P. Tardivel, Mélanges, 3 : XXXV.

64 Ibid.

65 J.-P. Tardivel, Mélanges, 3 : XXXVII.

66 J.-P. Tardivel, Mélanges, 3 : XXXVIII. 
mission de faire du journalisme catholique. Mais encore fallaitil se rendre à Rome et rencontrer le Pape.

En 1896, l'occasion s'offrit. Le premier congrès anti-maçonnique international devait avoir lieu, à Trente, à la fin de septembre. Tardivel résolut d'y assister, puis de se rendre à Rome, et “d'y rester jusqu'à ce que le Pape m'eût dit si je devais, oui ou non, continuer mon œuvre". ${ }^{67}$

Comme toujours, des amis généreux lui fournirent les moyens de faire ce nouveau voyage en Europe. Cette fois-ci, son grand vœu devait être réalisé: il rencontrait le Pape.

Enfin, c'est mon tour. Je me mets à genoux devant le Saint-Père, et Mgr de Nicole veut bien lui dire, en termes trop flatteurs, qui je suis et ce que je fais depuis quinze ans: “C'est un journaliste, Très Saint-Père, qui combat la franc-maçonnerie depuis de longues années, qui l'a bien combattue, puisqu'il s'est toujours appuyé sur vos enseignements, et il arrive de Trente oì il a pris part au congrès antimaçonnique.

En entendant parler de franc-maçonnerie et du Congrès, le Saint-Père, un peu affaissé, se redresse tout à coup et s'anime.

- Ah! dit-il, la franc-maçonnerie! il faut la combattre toujours, car cette secte est hostile à l'Eglise, qu'elle voudrait détruire; elle veut remplacer la religion de Notre-Seigneur par le naturalisme. Il faut la combattre, il faut la combattre! J'ai écrit des encycliques contre cette secte: "Humanum genus", et une lettre aux évêques d'Italie et au peuple italien. Les ave:z-vous lues?

- Très Saint-Père je les sais presque par cœur, tant je les ai lues et relues.

- Bien, très bien. Continuez à combattre la francmaçonnerie. Y a-t-il des francs-maçons au Canada?

- Il y en a, malheureusement, Très Saint-Père.

- Eh bien, combattez toujours! ${ }^{68}$

67 J.-P. Tardivel, Mélanges, 3 : XLII.

68 J.-P. Tardivel, Mélanges, 3 : XLIV. 
Tardivel venait de se voir confirmer sa mission de défenseur de l'orthodoxie, par le Pape.

Fort de cette approbation du Pape et de la position de la Vérité Tardivel continua à suivre le chemin où il avait marché jusque-là.

Toutefois, les épreuves n'étaient pas finies. La nouvelle occasion serait la question scolaire manitobaine qui se posa pendant la lutte électorale de 1896. Tardivel demandait, avec les évêques de la province de Québec et l'archevêque de SaintBoniface, une "loil remédiatrice" comme l'unique moyen de régler la difficulté.

Vers la fin de l'année 1897, Léon XIII exhortait les catholiques manitobains à ne pas refuser les satisfactions partielles. Tardivel n'hésita pas à entrer immédiatement dans la voie que traçait Léon XIII qui au mois d'octobre de la même année lui confirmait sa mission de journaliste catholique.

L'attitude de la Vérité "suscita une nouvelle tempête"."99 Dans ce contexte de lutte constante, dans ces conditions d'impression aussi difficiles, la santé de Tardivel devait s'altérer rapidement. À partir de 1903, la Vérité ne paraîtra plus régulièrement. Déjà son fondateur ne pouvait plus tenir le coup.

C'est vers cette époque, 15 octobre 1904, qu'Omer Héroux devient le rédacteur de la Vérité.

Lundi, le 24 avril 1905, J.-P. Tardivel mourait, à l'âge de 54 ans, dans sa demeure de Sainte-Foye.

MATHIEU GIRARD

${ }^{69}$ J.-P. Tardivel, Mélanges, 3 : LI. 